\title{
纳米集成电路互连线建模和光刻仿真中的大规模 并行计算方法
}

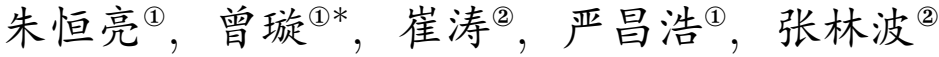 \\ (1) 复旦大学专用集成电路与系统国家重点实验室, 上海 201203 \\ (2) 中国科学院数学与系统科学研究院科学与工程计算国家重点实验室 (LSEC), 北京 100190 \\ * 通信作者. E-mail: xzeng@fudan.edu.cn
}

收稿日期: 2016-05-04；接受日期: 2016-08-04；网络出版日期: 2016-10-25

国家重点基础研究发展计划 (973 计划) (批准号: 2011CB309701, 2011CB309703)、国家高技术研究发展计划 (863 计划) (批准 号: 2012AA01A30901)、国家重点研发计划高性能计算重点专项项目 (批准号: 2016YFB0201304)、国家自然科学基金 (批准号: $61376040,61574046,91330201,61274032,91430215,91530323,11321061)$ 和中国科学院国家数学与交叉科学研究中心 (NC MIS) 资助项目

摘要集成电路规模庞大、结构复杂, 随着集成电路制造工艺进入纳米尺度, 复杂制造工艺中的工 艺波动严重影响电路性能, 给集成电路设计带来了巨大的挑战. 集成电路互连线建模与光刻仿真涉 及大规模 Maxwell 方程的数值求解, 计算复杂度高、规模庞大. 本文主要综述了在 973 项目资助下, 基于中国科学院科学与工程计算国家重点实验室陈志明教授提出的并行自适应有限元理论及大规 模并行计算平台 PHG 发展的纳米集成电路互连线建模和光刻仿真的大规模并行计算方法. 在集成 电路互连线建模方面, 一方面, 综述了寄生电容参数提取的并行自适应有限元方法 ParAFEMCap, 该 方法实现了可以在上百乃至上千 $\mathrm{CPU}$ 核上运行的并行寄生电容提取, 在 $1536 \mathrm{CPU}$ 核计算平台上达 到 $75.7 \%$ 左右的并行效率; 另一方面, 综述了一种结合边界元法和随机法的混合算法 BIE-WOS, 用于 导体或介质面电荷密度计算, 该方法具有随机法天然并行性的优势, 易于实现大规模并行计算, 本文 进一步在 5120 核计算平台上验证了算法近似线性的并行加速比. 在光刻仿真方面, 基于自适应有限 元计算框架 (PHG) 提出了集成电路光刻的并行自适应仿真方法, 采用各项异性的单轴完美匹配层方 法处理散射边界条件.

关键词 大规模集成电路 建模 寄生参数提取 光刻工艺 有限元方法 随机行走方法

\section{1 引言}

目前, 国际上集成电路制造工艺已经进入 $14 \mathrm{~nm}$ 节点, 芯片集成度达数十亿晶体管, 芯片面积 达数平方厘米, $\mathrm{CPU}$ 的时钟频率超过 $4 \mathrm{GHz}$. 根据国际半导体技术发展路线图 ITRS (international technology roadmap for semiconductors) 的预测, 集成电路制造工艺将在 2018 年进入 $7 \mathrm{~nm}$ 节点, 2020 年进入 $5 \mathrm{~nm}$ 节点. 集成电路中互连线宽度仅为几十纳米, 但是互连线层数可达十多层, 总长度可达数 
公里. 互连线的延时超过器件延时成为主宰芯片性能的关键因素. 集成电路制造工艺中的光刻工艺采 用 $193 \mathrm{~nm}$ 波长光源, 已远大于掩膜的特征尺寸, 光刻工艺进入了理论分辨率的极限. 大尺寸光波显著 的干涉和衍射效应造成了器件和互连线几何尺寸的严重畸变, 导致芯片成品率的大幅降低. 集成电路 的互连线建模分析以及光刻仿真对集成电路的性能分析和优化、成品率提升起着关键作用, 是指导纳 米尺度集成电路设计和制造的核心技术之一.

互连线是电路性能和可靠性的决定性因素, 包括串扰噪声、电源的网络电压降以及电磁耦合导致 的延迟偏差在内的信号完整性问题变得越来越严重. 这很大程度上是因为集成电路互连线结构十分 复杂、集成度增大导致互连线的寄生效应变得越来越严重. 随着集成电路工作速度递增, 特征尺寸的 进一步缩小, 以及铜互连材料的采用, 互连线寄生效应对电路性能的影响成为电路设计者必须考虑的 重要因素之一 ${ }^{[1,2]}$. 在芯片交付生产前, 从版图提取互连线的寄生参数, 进行考虑寄生效应的电路性 能分析和时序驱动的设计优化, 是集成电路设计中确保芯片性能的关键步骤. 互连线寄生参数提取 (parasitic extraction) 技术用于建立复杂结构互连线的等效电路模型 ${ }^{[3]}$, 从而对互连线的寄生效应建 立精确的描述. 精确的寄生参数提取方法是基于场求解器 (field solver), 即通过数值求解描述互连线 电学行为的物理方程 —Maxwell 方程组, 以获得互连线的等效 RLC 电路模型 [3]. 对于全芯片规模 庞大的互连线寄生参数提取, 工业界往往采用解析或半解析方法 ${ }^{[4]}$ 、基于模式匹配的全芯片提取方法, 以及 2 维或 2.5 维的场求解方法 ${ }^{2]}$ 等技术来简化求解, 而基于精确三维场求解器的寄生参数提取方 法仍是互连线建模的关键技术难题.

互连线寄生电容参数提取 (parasitic capacitance extraction) 的场求解器主要包括积分方程 (inte-

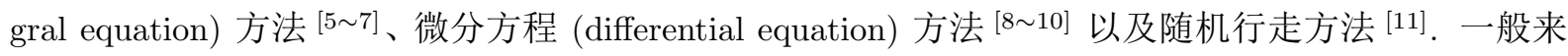
说, 积分方法基于边界元方法 (boundary-element method, BEM), 这类方法是将导体表面和介质层表 面离散成面元并且通过数值方法求解面元上的电荷量. 相比于微分方法, 积分方法的优点之一就是其 得到的线性方程组的维度要小得多. 然而, 因为 Green 函数 (Green's function) 具有空间全局性, 积分 方法的离散矩阵是稠密的, 以至于用直接法求解积分法的线性系统计算复杂度是 $O\left(N^{3}\right)$, 当使用迭代 法的时候, 计算复杂度可以降低至 $O\left(N^{2}\right)$, 因此需要采用加速算法, 如快速多极子方法 (fast multi-pole method, FMM) ${ }^{[5]}$ 、层次化方法 (hierarchical method) ${ }^{[6,7]}$ 等, 将计算复杂度进一步降低至 $O(N \log N)$. 微分方程方法包括有限差分法 ${ }^{[10]}$ 和有限元方法 ${ }^{[8,9]}$, 由于需要对计算区域进行离散, 因而相比于积分 方程方法, 需要求解较大规模的线性方程. 另一方面, 由于这类方法采用了具有局部性的基函数, 微分 方程方法得到的离散矩阵往往具有极高的稀疏性, 这十分有利于采用稀疏矩阵技术及并行化技术进行 快速求解. 此外, 随机行走方法 (floating random walk method) ${ }^{[11]}$, 采用 Monte Carlo 方法求解静电场 问题, 相比于积分方程方法和微分方程方法, 随机行走方法无需生成网格, 因此对内存要求极低, 在商 业工具中有着广泛的应用, 其主要问题在于数值精度和计算效率受限于 Monte Carlo 采样的收玫速度.

对于集成电路中复杂的互连线结构, 计算复杂度一直是互连线寄生参数提取的瓶颈问题. 对于大 规模互连线电路乃至全芯片寄生电容参数提取问题, 并行计算是解决互连线参数提取计算速度、内存 等瓶颈问题的有效途径之一. 现有的互连线寄生电容提取并行算法主要有基于并行边界元方法的电容

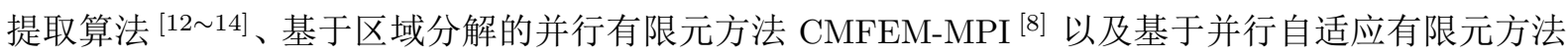
的 ParAFEMCap ${ }^{[9]}$. 文献 [13] 实现了 GPU 上的并行快速多极方法, 然而由于 GPU 上存储单元通常 比较小, 也成了该方法的瓶颈问题. 基于多核计算机系统并行电容提取方法 ${ }^{[8,12]}$ 在 $16 \mathrm{CPU}$ 核下实现 了并行计算, 但并行效率仅 57\%. 2016 年, 美国麻省理工大学 (MIT) Daniel 教授提出并行边界元算法 CAPLET ${ }^{[14]}$, 将并行规模扩展至约 800 个 CPU 核, 但并行效率急剧降至 $44 \%$.

集成电路互连线建模的另一个科学问题是导体和介质的面电荷密度计算问题 ${ }^{[15]}$, 随着集成电路 
特征尺寸和互连线间距不断缩小, 互连线导体和介质表面的电荷密度/电场强度不断上升, 为了防止电 介质被击穿, 需要准确计算导体和介质的面电荷密度. 与互连线寄生电容参数提取问题类似, 导体和 介质的面电荷密度计算通过数值方法求解电场的 Laplace 方程. 互连线寄生电容参数提取需要计算 的是每个导体的总电荷量, 而面电荷密度计算则需要计算导体和介质面上的电荷密度分布, 因此, 面 电荷计算具有更高的计算复杂度和计算精度要求. 一般而言, 广泛应用于寄生参数提取的有限差分、 有限元、边界元等数值解法也可以用于求解导体和介质的面电荷密度计算问题, 而随机行走方法通过 Monte Carlo 方法计算导体总电荷, 无法直接应用于导体和介质的面电荷密度计算.

另一方面, 进入 $32 \mathrm{~nm}$ 和 $22 \mathrm{~nm}$ 工艺节点后, 光刻机所采用的光源波长与掩膜的特征尺寸已达到 了同一个量级, 考虑掩膜和硅片表面三维形貌的三维电磁散射效应的精确光刻模型和仿真算法已列为 国际半导体技术发展路线图 ITRS 的前沿科学问题. 目前国际上已经提出了采用二维或三维 Maxwell 方程用于移相掩膜版的精确建模, 并提出了多种严格电磁分析方法如: 时域有限差分方法 (FDTD) ${ }^{[16] 、}$

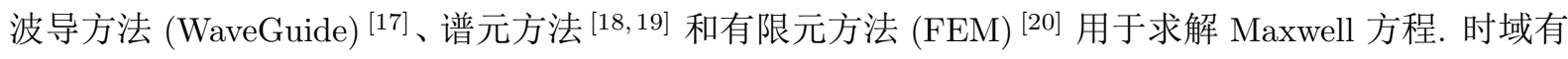
限差分方法 (FDTD) [16] 在时域求解 Maxwell 方程. 它对 Maxwell 方程在时间域和空间域进行差分离 散, 对得到的离散代数方程进行迭代求解. 为了保证时域有限差分方法的稳定性, 空间步长和时间步 长必须满足稳定性条件. 波导方法 (WaveGuide) ${ }^{[17]}$ 的主要思想是沿水平方向将掩膜版划分成一些薄 层, 使得每一个薄层中的介电常数在垂直方向是均一的. 在每一薄层中, 对 Maxwell 方程进行分离变 量可以得到一个特征值问题, 从而得到光在这一水平薄层中的电磁场传播模式. 进而在整个掩膜版的 上下加上合适的电场和磁场边界条件, 可以求得光通过掩膜版后反射波和透射波的分布. 广义特征振 荡谱元方法 $[18,19]$ 是一种可并行计算的移相掩膜版二维电磁快速仿真方法, 该方法沿垂直方向将掩膜 版划分成 $N$ 个垂直结构, 对若干种特征垂直掩膜结构, 采用谱元方法精确计算其电场的特征函数和特 征值, 并使用它们来表征任意一个垂直掩膜结构的电场分量; 在水平方向, 采用 Schwaz 迭代和并行计 算的技术求解 $N$ 个垂直掩膜结构的电场方程及边界条件. 该方法具有较好的并行效率, 但仅局限于求 解二维问题. 有限元方法求解给定频率下的时谐 Maxwell 方程, 文献 [20] 采用完美匹配层 (PML) 处 理边界条件. 有限元方法求解光刻仿真问题的主要难点在于完美匹配层边界条件的设置, 掩膜版分层 结构导致的驻波效应, 以及由此带来的计算精度和效率问题. 现有时域有限差分算法、波导方法、有限 元方法都存在着计算速度慢和收玫困难的问题, 不能适用于大规模问题的光刻模拟, 工业界仍缺少一 套高效、成熟的三维光刻工艺仿真工具.

集成电路互连线建模和光刻工艺仿真涉及大规模复杂介质环境 Maxwell 方程组的数值求解, 互连 线寄生电容参数提取、导体和介质面电荷计算的核心科学问题是求解静电场假设 Maxwell 方程组, 光 刻工艺仿真的核心科学问题则是求解全波 Maxwell 方程组. 这 3 个问题的计算复杂度高、规模庞大, 发展大规模并行计算方法是解决计算瓶颈问题的有效途径.

本文主要综述了在 973 项目资助下, 基于中国科学院科学与工程计算国家重点实验室陈志明教授 提出的并行自适应有限元理论 ${ }^{[21,22]}$ 及大规模并行计算平台 $\mathrm{PHG}{ }^{1)}$ 发展的纳米集成电路互连线建模 和光刻仿真的大规模并行计算方法. 在互连线寄生参数提取方面, 首先综述了寄生电容参数提取的并 行自适应有限元方法 ParAFEMCap ${ }^{[9]}$, 该方法实现了基于业界标准 GDSII 版图的、可以在上百乃至 上千 CPU 核上运行的并行寄生电容提取, 具有极高的并行效率和计算精度的优点, 在 $1536 \mathrm{CPU}$ 核下 该方法仍具有 $75.7 \%$ 的并行效率. 并行自适应有限元方法也应用在了互连线寄生阻抗参数提取, 实现 了并行寄生阻抗提取工具 ParAFEMImp ${ }^{[22,23]}$, 在 $6144 \mathrm{CPU}$ 核下具有 $84 \%$ 左右的并行效率, 在 12288 CPU 核下仍具有 $54 \%$ 左右的并行效率. 在导体和介质的面电荷密度计算方面, 综述了一种结合边界

1) PHG, Parallel Hierarchical Grid. URL:http://lsec.cc.ac.cn/phg/. 
元法和随机行走方法的混合算法 BIE-WOS (boundary integral equation-walk on spheres) ${ }^{[15]}$. 它可针 对金属和介质组成的粗䊁面, 采用统一方法进行处理. 其核心想法是构建一个虚拟半球面与待求的局 部边界相交, 形成一个封闭曲面; 半球面上采用 WOS 等随机法得到未知电位; 然后对封闭曲面采用特 殊 Green 函数的边界元法进行求解. 它具有随机行走方法天然并行性的优势, 易于实现大规模并行计 算, 本文进一步在 5120 核计算平台上验证了 BIE-WOS 方法近似线性的并行加速比. 在光刻仿真方面, 针对电磁散射问题的有限元求解方法, 采用各向异性的单轴完美匹配层方法处理散射边界条件; 基于 自适应有限元计算框架, 设计自适应数值算法; 通过后验误差估计决定完全匹配层的厚度和其中的材 料参数, 在 PML 层内解变化缓慢的区域自动产生较粗的网格, 使得总计算量对于层的厚度不敏感; 从 而在并行自适应有限元平台 $(\mathrm{PHG})$ 实现了集成电路光刻的并行自适应仿真.

本文在第 2 和 3 节将分别介绍互连线寄生电容提取的并行自适应有限元方法以及结合边界元和 随机行走的表面电荷计算方法; 在第 4 节将介绍基于有限元的光刻工艺仿真算法; 第 5 节对大规模高 性能计算机集群并行计算做了讨论与分析; 第 6 节给出总结.

\section{2 基于并行自适应有限元方法的互连线寄生电容参数提取方法}

在互连线寄生参数提取方面, 将并行自适应有限元方法应用于寄生电容参数提取, 实现了基于业 界标准 GDSII 版图的, 可以在上百乃至上千 CPU 核上运行的并行寄生电容提取工具 ParAFEMCap ${ }^{[9]}$, 本节介绍其具体模型及算法.

\section{1 基于有限元的寄生电容参数提取}

集成电路互连线为多层介质复杂结构的多导体结构, 互连线寄生电容矩阵可用静电场方法计算. 互连线多导体系统中的每一个导体, 遵循静电场中的静电平衡性质, 即导体内部的场强为零, 导体是 一个等势体, 导体所带净电荷以面电荷的形式分布在导体表面, 导体内部不存在净电荷. 因此, 对于包 含 $N$ 个导体的互连线系统, 其导体电势 $\phi_{1}, \phi_{2}, \ldots, \phi_{N}$ 和导体表面净电荷 $q_{1}, q_{2}, \ldots, q_{N}$ 满足关系式:

$$
\begin{aligned}
q_{1} & =C_{11} \phi_{1}+C_{12} \phi_{2}+\cdots+C_{1 N} \phi_{N}, \\
q_{2} & =C_{21} \phi_{1}+C_{22} \phi_{2}+\cdots+C_{2 N} \phi_{N}, \\
& \vdots \\
q_{N} & =C_{N 1} \phi_{1}+C_{N 2} \phi_{2}+\cdots+C_{N N} \phi_{N},
\end{aligned}
$$

其中, $[C]$ 是 $N \times N$ 的电容矩阵, $C_{i i}$ 表示导体 $i$ 自电容, $C_{i j}, i \neq j$ 为导体 $i$ 和导体 $j$ 之间的互容.

有限元方法电容提取归结为求解给定边界条件的空间电势分布的 Laplace 方程问题:

$$
-\nabla \cdot \varepsilon \nabla \phi(\boldsymbol{r})=0 \text { in } \Omega
$$

边界条件:

$$
\left\{\begin{array}{l}
\phi(\boldsymbol{r})=\phi_{\mathrm{c}} \text { on } \Gamma, \\
\phi(\boldsymbol{r})=0 \text { at } \infty,
\end{array}\right.
$$

其中 $\Omega$ 为所有介质层计算区域, $\Gamma$ 为导体表面, $\phi_{\mathrm{c}}$ 为导体表面电势, 其值取决于导体是置为 $1 \mathrm{~V}$ 或者 置 $0 \mathrm{~V}, \varepsilon$ 为介质层介电常数. 在有限元方法中, 应用四面体网格进行空间离散, 在每一个四面体单元 


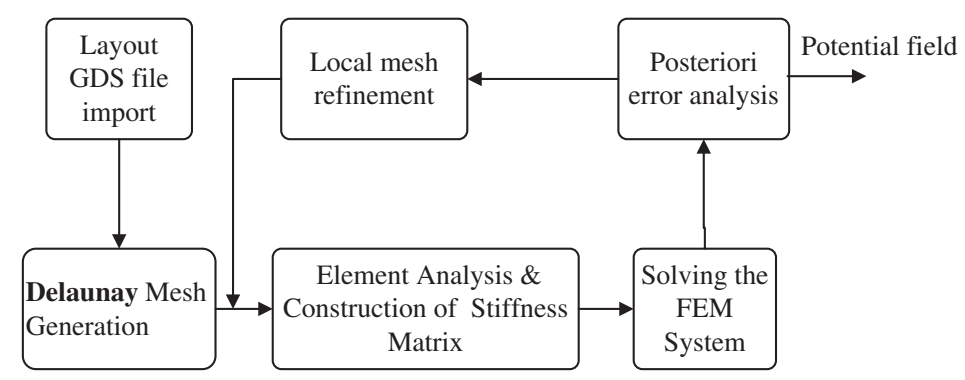

图 1 ParAFEMCap 中的自适应网格划分基本框图 ${ }^{[9]}$

Figure 1 Adaptive meshing in ParAFEMCap ${ }^{[9]}$

中, 电势 $\phi(r)$ 可以离散为一组基函数展开:

$$
\phi^{e}(\boldsymbol{r})=\sum_{k=1}^{n} \phi_{k}^{e}(\boldsymbol{r}) \mathfrak{L}_{k}^{e}(\boldsymbol{r}),
$$

其中上标 $e$ 表示四面体单元 $e$ 局部量, $\phi_{k}^{e}(\boldsymbol{r})$ 在四面体单元 $e$ 节点 (顶点、边、面、体) 上的未知量, $\mathfrak{L}_{k}^{e}(\boldsymbol{r})$ 为一组定义在四面体单元的节点的多项式基函数, $n$ 为四面体单元基函数的数目.

通过组合所有四面体单元的局部展开, Laplace 方程被离散为

$$
[\boldsymbol{K}] \cdot \boldsymbol{\phi}=\boldsymbol{b},
$$

其中, $[\boldsymbol{K}]$ 为总体刚度矩阵, 它是通过组装所有四面体单元的局部刚度矩阵 $\left[\boldsymbol{K}^{e}\right]$ 而成, 单元局部刚度 矩阵 $\left[\boldsymbol{K}^{e}\right]$ 可表示为

$$
K_{k l}^{e}=\int_{\Omega^{e}} \varepsilon^{e} \nabla \mathcal{L}_{k}^{e}(\boldsymbol{r}) \nabla \mathcal{L}_{l}^{e}(\boldsymbol{r}) \mathrm{d} \Omega^{e} .
$$

通过进一步施加边界条件, 可以通过采用稀疏矩阵数值技术求解代数方程获得静电场分布 $\phi$.

在第 $i$ 与第 $j$ 个导体施加电压 $1 \mathrm{~V}$, 其余导体置电压 $0 \mathrm{~V}$ 时, 通过有限元方法可以计算出系统的 总静电能 $W_{i j}{ }^{[9]}$ :

$$
W_{i j}=\frac{1}{2} \int_{\Omega} \varepsilon \nabla \phi \cdot \nabla \phi \mathrm{d} \Omega=\frac{1}{2} \sum_{i=1}^{N} \int_{\Omega_{e}} \varepsilon^{e} \nabla \phi^{e} \cdot \nabla \phi^{e} \mathrm{~d} \Omega^{e} .
$$

通过系统总静电能 (7) 计算电容值的方法如下:

(1) 当设置第 $i$ 个导体电压为 $1 \mathrm{~V}$, 其他导体电压为 $0 \mathrm{~V}$, 由式 (1) 可知 $q_{i}=C_{i i}$, 因此

$$
C_{i i}=2 \cdot W_{i i} .
$$

(2) 同时置导体 $i, j$ 电压为 $1 \mathrm{~V}(i \neq j)$, 其余导体电压为 $0 \mathrm{~V}$, 可以得到

$$
C_{i j}=W_{i j}-\frac{C_{i i}+C_{j j}}{2} \text {. }
$$

\section{2 并行自适应有限元提取工具 ParAFEMCap}

并行自适应有限元电容提取工具 ParAFEMCap ${ }^{[9]}$ 采用局部加密的协调四面体网格以及后验误 差估计实现了有限元网格的自适应加密策略, 其基本框图如图 1 所示. ParAFEMCap 输入互连线的 版图, 生成互连线几何结构的初始的 Delaunay 四面体网格, 采用 “组装 $\Rightarrow$ 求解 $\Rightarrow$ 估计和标记 $\Rightarrow$ 加 密” 的自适应循环策略对网格进行自动加密进而求解静电场问题. 自适应加密中的核心步骤如算法 1 所示. 


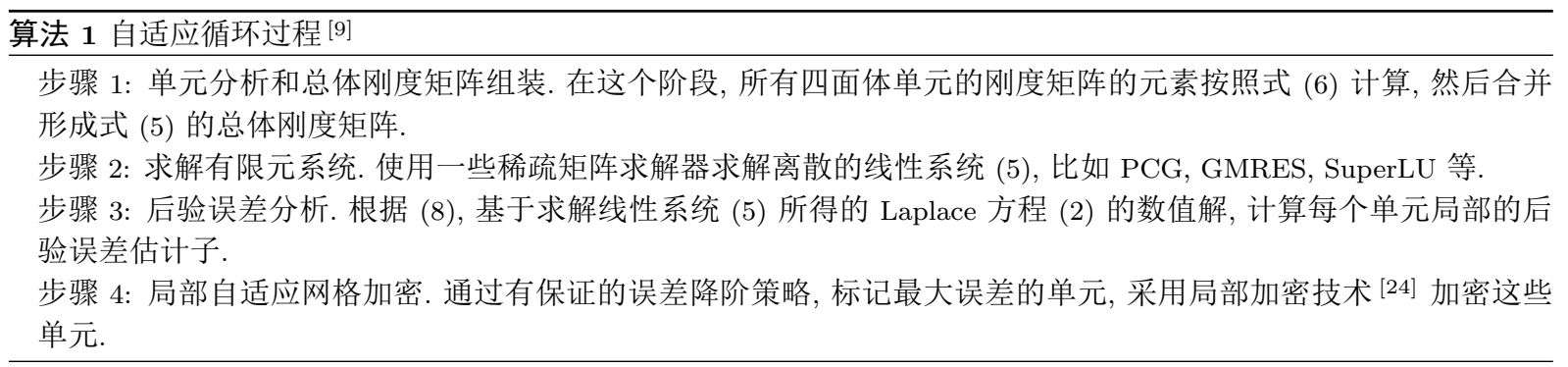

\subsection{1 后验误差估计}

后验误差估计用于确定哪些区域需要更精细网格以解决电势奇异性的问题. 令 $\phi_{h}$ 为方程 (2) 解 $\phi$ 的有限元近似, 每个四面体单元 $e$ 的残量型后验误差估计子 $\eta_{e}$ 定义如下:

$$
\eta_{e}^{2}=h_{e}^{2}\left\|\varepsilon \Delta \phi_{h}\right\|_{0, e}^{2}+\sum_{f \in F(e), f \subset \Omega} h_{f}\left\|\left[\varepsilon \nabla \phi_{h} \cdot \boldsymbol{n}_{f}\right]_{f}\right\|_{0, f}^{2},
$$

其中, $h_{e}$ 代表单元 $e$ 的直径, $F(e)$ 为单元 $e$ 所有面的集合, $h_{f}$ 为面 $f$ 的直径, $\boldsymbol{n}_{f}$ 为向外指向面 $f$ 的 单位法向向量, $\Omega$ 为整个计算区域. 在式 (8) 中定义的后验误差估计子由两部分组成, 第 1 部分与方程 (2) 的残量相关, 第 2 部分代表了穿越单元 $e$ 的表面的电场或者电位矢量的非连续性. 根据定理 1 , 后 验误差估计子 $\eta_{e}$ 可以用于估计真实电势误差的上下界.

定理1 存在只依赖网格 $M$ 最小角的常数 $C_{1}$ 和 $C_{2}$, 使得对于任意的单元 $e \in M$, 有

$$
\left\|\phi-\phi_{h}\right\|_{1} \leqslant C_{1}\left(\sum_{e \in M} \eta_{e}^{2}\right)^{1 / 2}, \quad C_{2} \eta_{e}^{2} \leqslant\left\|\phi-\phi_{h}\right\|_{1, w_{e}}^{2},
$$

其中, $w_{e}$ 为包含单元 $e$ 以及所有与 $e$ 共面的单元的集合 ${ }^{[21]}$.

根据定理 1 , 自适应方法具有最优的收玫速率特性, 能量误差按照理论最优的 $O\left(N^{-p / 3}\right)$ 的方式 衰减, 衰减速度随着自由度数目 $N$ 和基函数的阶数 $p$ 增加而加快.

\subsection{2 网格局部加密}

根据后验误差估计的结果, 可以定义全局后验误差估计 $\eta_{M}$ 和最大的单元后验误差估计子 $\eta_{e}^{\max }$ 为

$$
\eta_{M}=\left(\sum_{e \in M} \eta_{e}^{2}\right)^{1 / 2}, \quad \eta_{e}^{\max }=\max _{e \in M} \eta_{e}
$$

自适应有限元根据后验误差估计子采用最大误差策略 (maximum strategy) 来调整网格, 即将满足 $\eta_{e}>$ $\theta \eta_{e}^{\max }$ 的单元 $e$ 标记为待加密单元, 其中 $\theta$ 为细化比例.

ParAFEMCap 采用二分加密的方法 [24] 对标记好的网格单元进行局部加密, 其优点在于能在不引 入太多不必要的网格单元的前提下, 保证在有限步内产生协调的加密网格.

\subsection{3 并行实现}

在 ParAFEMCap 中, 采用 MPI 和 OpenMP 混合并行的编程模式, 即通过 OpenMP 实现同一计 算节点同一进程内不同线程的并行化, 通过 MPI 实现不同进程间通讯, 可以实现数百上千个 CPU 核 
(a)

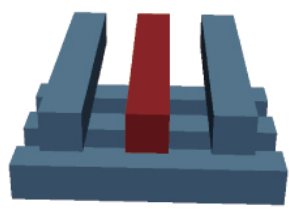

(b)
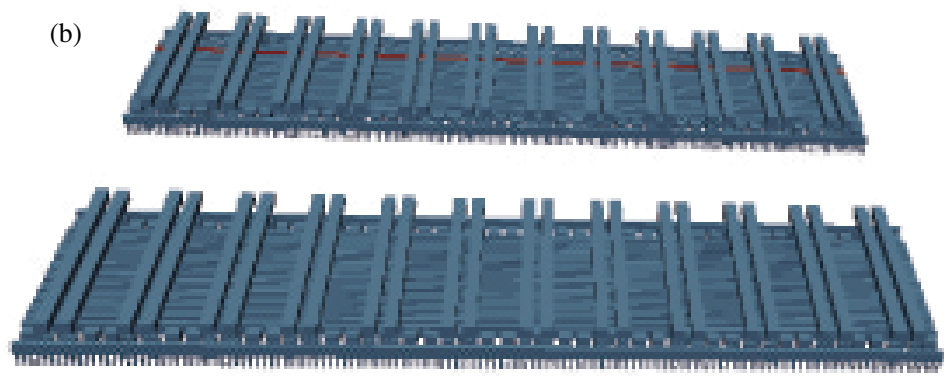

图 2 (网络版彩图) 互连线算例

Figure 2 (Color online) Test structure. (a) $3 \times 3$ bus; (b) EE-Array interconnect ${ }^{[9]}$

表 $13 \times 3$ 总线算例电容值 $(\mathrm{fF})^{[9]}$

Table 1 The capacitances of $3 \times 3$ bus test case (fF) ${ }^{[9]}$

\begin{tabular}{cccccc}
\hline & $C_{1,1}$ & $C_{2,2}$ & $C_{3,3}$ & $C_{1,2}$ & $C_{1,6}$ \\
\hline FastCap & 1.2825 & 1.473 & 1.283 & -0.4302 & -0.1899 \\
HiCap & 1.2838 & 1.475 & 1.284 & -0.4296 & -0.1902 \\
ParAFEMCap & 1.313 & 1.4902 & 1.3084 & -0.4341 & -0.1865 \\
\hline
\end{tabular}

的高度并行化的电容提取算法. 进一步采用分布式的网格和刚度矩阵的存储方式, 并通过 Hibert 曲线 和层次二叉树进行网格划分, 采用动态负载平衡技术保证不同计算节点上的负载均衡, 采用并行的线 性方程迭代求解器, 从而实现较高的并行可扩展性 [9].

\section{3 数值结果}

基于并行自适应有限元的互连线电容提取算法 ParAFEMCap 采用并行自适应有限元平台 Parallel Hierarchical Grid (PHG), 并且采用 $\mathrm{C} / \mathrm{C}++$ 编程语言实现. 实验计算采用如图 2 所示的 $3 \times 3$ 互连总 线结构以及存储器单元的互连结构, 在国家超算天津中心的天河 $1 \mathrm{~A}$ 并行计算机对 ParAFEMCap 工 具进行精度和并行可扩展性测试.

\subsection{1 精度分析及最优收敛特性}

ParAFEMCap 互连线寄生电容提取算法采用后验误差估计和自适应网格加密, 在理论上具有很 高的数值精度. 首先, 采用 $3 \times 3$ 总线算例来验证 ParAFEMCap 的精度. 表 1 给出了 ParAFEMCap 与 MIT 大学提出的基于边界元方法的电容提取工具 FastCap ${ }^{[5]}$ 和 TAMU 大学 HiCap ${ }^{[7]}$ 的比较结 果. FastCap 方法和 HiCap 方法的离散面元数目分别为 97488 和 110592, ParAFEMCap 方法的自由 度 (DoFs) 数目为 87400 . 实验结果可以看出 ParAFEMCap 的结果和 FastCap 和 HiCap 的结果基本 吻合, 差异小于 $2.3 \%$.

图 3 给出了 ParAFEMCap 的误差收玫曲线, 其中 P1 与 P2 分别对应选取一阶基函数与二阶基函 数得到的收玫曲线. 图中带菱形和方块的四条曲线中, 两条实线分别表示计算电容值 $C_{22}$ 时使用一阶基 函数和二阶基函数的后验误差收玫曲线, 两条虚线表示理论的最优收敛曲线. 可以看出, ParAFEMCap 的后验误差按照 $O\left(N^{-p / 3}\right)$ 的方式下降, 其中 $p$ 为使用的有限元基函数的阶数, 从而验证了自适应加 密的最优收敛特性. 


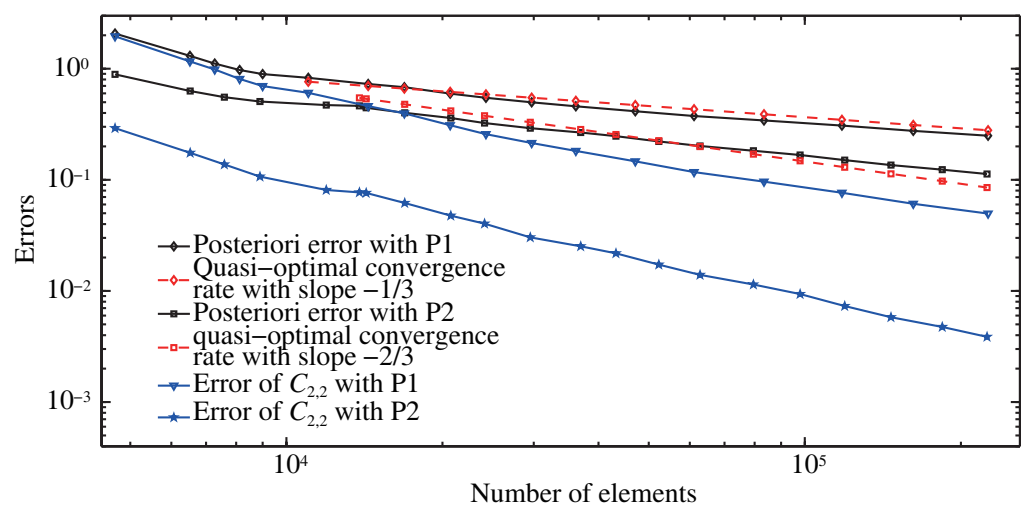

图 3 (网络版彩图) ParAFEMCap 收敛性分析 ${ }^{[9]}$

Figure 3 (Color online) Convergence of ParAFEMCap ${ }^{[9]}$

表 2 存储单元阵列互连线 MPI 并行可扩展性测试 [9]

Table 2 Parallel scalability of OpenMP in EE-Array test case ${ }^{[9]}$

\begin{tabular}{ccccc}
\hline \#MPI threads & \#CPU cores & Computation time (s) & Speedup & Parallel efficiency (\%) \\
\hline 2 & $2 \times 6=12$ & 368.4 & $1 \mathrm{X}$ & 100 \\
8 & $8 \times 6=48$ & 115.5 & $3.19 \mathrm{X}$ & 79.94 \\
16 & $16 \times 6=96$ & 55.1 & $6.69 \mathrm{X}$ & 83.58 \\
32 & $32 \times 6=192$ & 27.7 & $13.30 \mathrm{X}$ & 83.12 \\
64 & $64 \times 6=384$ & 13.5 & $27.29 \mathrm{X}$ & 85.28 \\
128 & $128 \times 6=768$ & 7.1 & $51.89 \mathrm{X}$ & 81.07 \\
256 & $256 \times 6=1536$ & 3.8 & $96.95 \mathrm{X}$ & 75.74 \\
\hline
\end{tabular}

图中带。和 $\times$ 的两条曲线分别表示在使用一阶基函数和二阶基函数情况下电容值 $C_{22}$ 的误差收 玫曲线. 由于电容的计算需要进行电势的积分, 这进一步消除由于有限元数值计算带来的误差, 使得 ParAFEMCap 得到的电容值的误差按照比 $O\left(N^{-p / 3}\right)$ 更快的方式下降.

\subsection{2 并行效率分析}

表 2 给出了存储单元阵列 EE-Array 互连线 (图 2(b)) 的 MPI 并行效率, 该算例包含 2232 根导 线, 22464 个通孔, 自由度为 10645101. 在算法测试中, 每个 MPI 进程拥有 6 个 OpenMP 线程, 每个线 程运行在一个 $\mathrm{CPU}$ 核上. 由表 2 可以看出, 当 MPI 进程数为 $16, \mathrm{CPU}$ 核数为 96 时, 并行效率高于 $83 \%$. 即便当 MPI 进程数目为 256 , CPU 核数达到 1536 时, 并行效率仍然高达 $75 \%$. 数值结果验证了 并行自适应有限元算法 ParAFEMCap 对于求解大规模互连线电容提取问题具有良好的并行扩展性.

\section{3 一种结合边界元方法和随机方法的并行面电荷密度计算方法}

随着集成电路特征尺寸不断缩小, 互连线间距不断缩小, 互连线导体和介质表面的电荷密度/电场 强度不断上升. 为了防止电介质被击穿, 需要准确计算导体和介质的面电荷密度. 数学上, 集成电路寄 生电容参数提取和表面面电荷密度计算都可归结为第一类边界条件下 (Dirichlet boundary condition) 


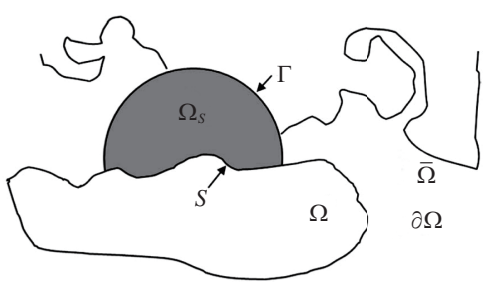

图 4 BIE-WOS 算法原理图 [15]

Figure 4 Illustration of BIE-WOS method ${ }^{[15]}$

Laplace 方程求解. 互连线寄生电容参数提取需要计算的是每个导体的总电荷量, 而面电荷密度计算 则需要计算导体和介质面上的电荷密度分布, 因此, 面电荷计算具有更高的计算复杂度和计算精度 要求.

互连线寄生电容参数提取的场求解器可大致分为确定性方法和随机方法, 确定性方法包括有限差 分方法、有限元方法和边界元方法等, 需要对计算区域进行离散、全局求解; 随机方法即随机行走方 法, 是基于 Feynman-Kac 定理的一个具体应用, 它将电位 (或面电荷密度) 表示为区域边界电位值的 某种加权平均 ${ }^{[25]}$, 可有效计算全芯片中局部区域的解而无需得到整个芯片的场分布, 因此极易实现大 规模并行. 但随机行走方法无法直接应用于表面面电荷密度计算问题. 一种较为特殊的 Last-passage 随机方法 [26] 可以计算导体表面的面电荷密度, 但它仅适用于 Dirichlet 边界条件为常数的导体平面, 而无法处理介质平面的面电荷密度提取问题.

针对集成电路面电荷密度计算问题, 我们提出了一种结合边界元方法和随机方法的混合算法 BIEWOS (boundary integral equation-walk on spheres) ${ }^{[15]}$. 它可针对金属和介质组成的粗粘面, 采用统一 的方法进行处理. BIE-WOS 方法的特点有: (1) 它属于一种局部性解法, 可提取局部边界上的面电荷 密度, 而无需进行全局求解; (2) 尽管它仍需对局部边界表面进行离散, 建立线性方程组并求解, 但它 无需对全芯片内所有导体和介质边界进行全局离散, 内存开销小; (3) 仍具有随机方法天然并行性的 优势, 易于实现大规模并行计算. BIE-WOS 方法结合了边界元方法和随机方法的优点. 与随机方法相 比, 它可直接计算边界上的面电荷密度这类 Neumann 值, 实现了完全意义上的场求解, 极大地扩展了 随机方法的适用范围. 与边界元方法相比, 它仍具有随机方法的局部性解法、内存开销小、易于并行等 优点.

\subsection{BIE-WOS 算法的基本原理}

BIE-WOS 算法求解集成电路中导体表面面电荷密度的算法原理如图 4 所示. 在开区域 $\bar{\Omega}$ 内电位 $u$ 满足 Laplace 方程, 区域边界 $\partial \Omega$ 为粗精曲面, 边界材料为导体、介质或它们的组合, 边界 $\partial \Omega$ 上的 电位分布函数 $u_{0}(\boldsymbol{r})$ 已知, 如式 (10) 所示:

$$
\left\{\begin{array}{l}
\nabla^{2} u(\boldsymbol{r})=0, \quad \boldsymbol{r} \in \bar{\Omega} \\
u(\boldsymbol{r})=u_{0}(\boldsymbol{r}), \quad \boldsymbol{r} \in \partial \Omega,
\end{array}\right.
$$

需求解边界 $\partial \Omega$ 上的面电荷密度, 即 $\frac{\partial u}{\partial \boldsymbol{n}}$, 其中 $\boldsymbol{n}$ 是区域 $\Omega$ 的单位外法向量.

BIE-WOS 算法结合了边界元方法和随机方法的特点. 首先, 它构造一个半径为 $a$ 的球体 $S_{a}$, 如 图 4 所示, 球体与粗䊁边界相交得到一个 patch 曲面 $S$, 假设 patch 曲面 $S$ 包含待求面电荷密度的 边界; 球体在区域外的半球面 $\Gamma$ 和边界上的粗䊅 patch 曲面 $S$ 构成一个封闭区域 $\Omega_{S}$, 其边界为曲面 $\Gamma \cup S$; 在封闭曲面 $\Gamma \cup S$ 上应用球面两点镜像 Green 函数边界元方法进行求解, 其中: 半球面 $\Gamma$ 积分 
中包含的未知电位 $u\left(r^{\prime}\right)$ 可以通过 WOS 等随机方法计算得到, 而粗粘 patch 曲面上的积分包含超奇 异积分, 它可以通过一定的数值技巧进行正则化, 降低奇异性; 最后, 求解线性方程组后可得到待求边 界上的面电荷密度.

在封闭区域 $\Omega_{S}$ 的边界 $\Gamma \cup S$, 若选择 Green 函数 $G\left(\boldsymbol{r}, \boldsymbol{r}^{\prime}\right)$ 为半径为 $a$ 的球体区域 $S_{a}$ 在齐次边 界下的 Green 函数, 并采用第一类边界积分方程归化的边界元方法求解, 可得到如下边界积分方程:

$$
u(\boldsymbol{r})=-\int_{\Gamma} \frac{\partial G\left(\boldsymbol{r}, \boldsymbol{r}^{\prime}\right)}{\partial \boldsymbol{n}_{\boldsymbol{r}^{\prime}}} u\left(\boldsymbol{r}^{\prime}\right) \mathrm{d} s_{\boldsymbol{r}^{\prime}}+\int_{S}\left[-\frac{\partial G\left(\boldsymbol{r}, \boldsymbol{r}^{\prime}\right)}{\partial \boldsymbol{n}_{\boldsymbol{r}^{\prime}}} u\left(\boldsymbol{r}^{\prime}\right)+G\left(\boldsymbol{r}, \boldsymbol{r}^{\prime}\right) \frac{\partial u\left(\boldsymbol{r}^{\prime}\right)}{\partial \boldsymbol{n}_{\boldsymbol{r}^{\prime}}}\right] \mathrm{d} s_{\boldsymbol{r}^{\prime}}, \quad \boldsymbol{r} \in \Omega_{S},
$$

其中, $u(\boldsymbol{r})$ 和 $u\left(\boldsymbol{r}^{\prime}\right)$ 分别为 $\boldsymbol{r}$ 点和 $\boldsymbol{r}^{\prime}$ 点的电位, $\boldsymbol{n}_{\boldsymbol{r}^{\prime}}$ 为 $\boldsymbol{r}^{\prime}$ 点的单位外法向量.

在式 (11) 中, 上半球面 $\Gamma$ 的积分中仅包含电位 $u\left(\boldsymbol{r}^{\prime}\right)$, 而不含电位的法向导数 $\frac{\partial u\left(\boldsymbol{r}^{\prime}\right)}{\partial \boldsymbol{n}_{\boldsymbol{r}^{\prime}}}$, 其中电位 $u\left(\boldsymbol{r}^{\prime}\right)$ 可以通过 WOS 等随机方法计算得到.

利用点配置边界元方法 (collocation BEM) 离散边界积分方程 (11) 得到线性方程组为

$$
K\left[\frac{\partial u}{\partial \boldsymbol{n}}\right](\boldsymbol{r})=b(\boldsymbol{r}), \quad \boldsymbol{r} \in S,
$$

其中,

$$
\begin{gathered}
K\left[\frac{\partial u}{\partial \boldsymbol{n}}\right] \equiv \int_{S} G\left(\boldsymbol{r}, \boldsymbol{r}^{\prime}\right) \frac{\partial u\left(\boldsymbol{r}^{\prime}\right)}{\partial \boldsymbol{n}_{\boldsymbol{r}^{\prime}}} \mathrm{d} s_{\boldsymbol{r}^{\prime}} \\
b(\boldsymbol{r}) \equiv\left[\frac{u(\boldsymbol{r})}{2}+\text { p.v. } \int_{S} \frac{\partial G\left(\boldsymbol{r}, \boldsymbol{r}^{\prime}\right)}{\partial \boldsymbol{n}_{\boldsymbol{r}^{\prime}}} u\left(\boldsymbol{r}^{\prime}\right) \mathrm{d} s_{\boldsymbol{r}^{\prime}}\right]+\int_{\Gamma} \frac{\partial G\left(\boldsymbol{r}, \boldsymbol{r}^{\prime}\right)}{\partial \boldsymbol{n}_{\boldsymbol{r}^{\prime}}} u\left(\boldsymbol{r}^{\prime}\right) \mathrm{d} s_{\boldsymbol{r}^{\prime}} .
\end{gathered}
$$

这里 p.v. 代表双层位势 (double layer potential) 的 Cauchy 主值积分 (Cauchy principal value); 其中, 面元内的普通积分采用 Gauss 积分, 对于弱奇异 (weak singularities) 和强奇异 (strong singularities) 积 分, 采用极坐标转换等数值技术转化为普通积分.

若待求面电荷密度的表面为整个区域的边界 $\partial \Omega$, 可构造一系列的小球体, 使其与区域边界 $\partial \Omega$ 相 交得到 patch 面; 所有的 patch 面覆盖全部待求边界 $\partial \Omega$. 在每个 patch 面上, 可并行、独立地进行曲 面离散、数值积分、建立线性方程组并求解. 在 BIE-WOS 算法中, 由于每个 patch 面间完全独立地并 行计算, 没有数据交换, 容易实现极高的并行度. 而在每个 patch 面内部, 采用随机方法计算球面上点 的电位，也极易实现高度并行化. 这种 patch 面之间的独立性和 patch 面内部随机方法的并行性, 是 并行 BIE-WOS 算法在大规模计算平台下获得高并行度的关键.

\section{2 实验结果}

集成电路中的互连线导体和介质表面的电荷密度过高会导致电介质被击穿. 为此, 需要准确计算 导体和介质的面电荷密度. 在实验中, 通过一个算例来模拟电荷周围区域的电荷密度分布情况. 利用 OpenMP 和 MPI 并行化技术, 采用 $\mathrm{C} / \mathrm{C}++$ 语言实现了并行 BIE-WOS 算法, 在上海交通大学超级计 算机 $\pi$ 上验证并行 BIE-WOS 算法的精度和并行效率. 为简化起见, 以下半径、距离、电荷量都进行 了归一化处理.

图 5 在点 $(2,0,0)$ 处有一个电荷量 $q=1$ 的点电荷, 需要计算一个半径 $R=5$ 的大球面上的面电 荷密度分布. 例如, 在点 $o=(0,0,5)$ 处构建一个 $a=1$ 的小球面, 计算其附近的面电荷密度分布, 需要 离散大球面上的 patch 面, 离散后的面元如图 5(b) 所示. 构建大量的小球面, 使其 patch 面覆盖所有 大球表面后, 利用并行计算可得到大球面上所有点的面电荷密度, 其相对误差分布如图 6 所示. 

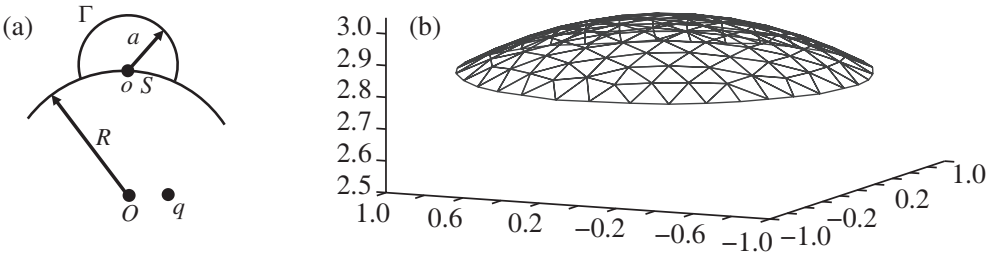

图 5 大球面的面电荷密度计算示意图 ${ }^{[15]}$

Figure 5 Illustration of solving the charge density on a big sphere ${ }^{[15]}$. (a) The schematic of the big sphere case with a hemisphere; (b) the discretization of the patch surface on the big sphere

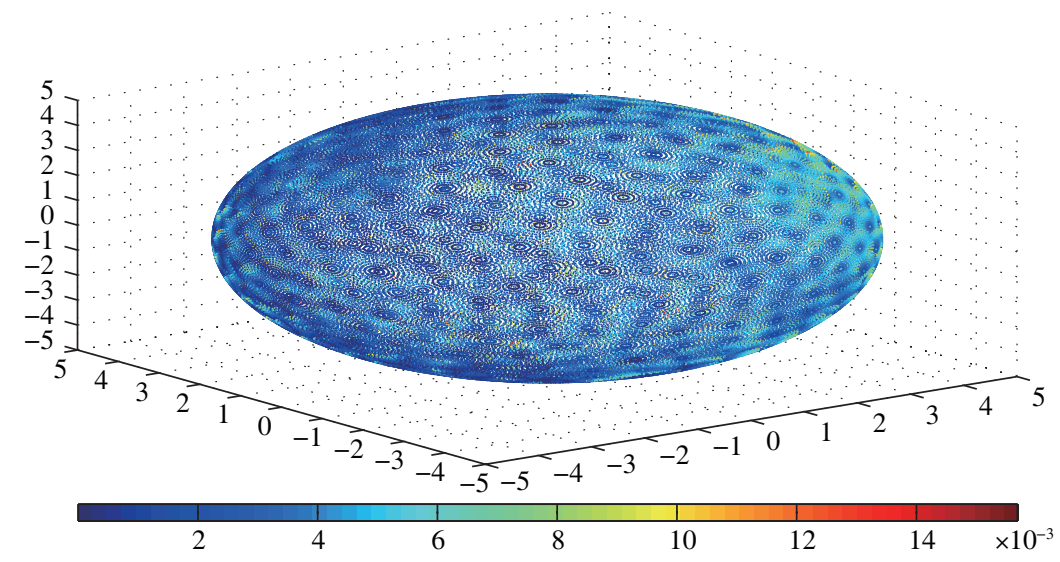

图 6 (网络版彩图) 大球面的面电荷密度相对误差分布图

Figure 6 (Color online) Relative error distribution of the charge density on the big sphere

表 3 求解大球面面电荷密度的并行效率

Table 3 Accuracy and scalability of charge density on a big sphere

\begin{tabular}{ccccccc}
\hline Load & \#Nodes & \#CPU cores & Time & Speedup & Efficiency (\%) & Max error (\%) \\
\hline $1 \mathrm{x}$ & 1 & 16 & 201.94 & 1 & 100.0 & 1.05 \\
$40 \mathrm{x}$ & 40 & 640 & 222.52 & 40 & 100.0 & 1.35 \\
$80 \mathrm{x}$ & 80 & 1280 & 207.19 & 85.9 & 106.0 & 1.35 \\
$160 \mathrm{x}$ & 160 & 2560 & 203.34 & 175.1 & 96.0 & 1.46 \\
$240 \mathrm{x}$ & 240 & 3840 & 229.89 & 232.3 & 95.0 & 1.46 \\
$320 \mathrm{x}$ & 320 & 5120 & 234.18 & 304.1 & & 1.46 \\
\hline
\end{tabular}

表 3 进一步给出了算法弱可扩展性的验证结果, 在不同数目的计算节点下采用等比例的任务负 载, 验证了算法的并行效率和精度. 表中最后一列给出的误差是指在对应的 $N$ 个任务负载的计算结果 相对于解析解的最大相对误差, 不同任务负载计算结果的最大相对误差均小于 $1.46 \%$. 算法的并行加 速比如图 7 所示, 我们使用了 320 个计算节点, 每个计算节点包含 16 个 $\mathrm{CPU}$ 核, 共计 5120 个核. 在 5120 个核上, 并行 BIE-WOS 算法的加速效率为 $95 \%$, 验证了该算法预期中的近似线性加速比. 


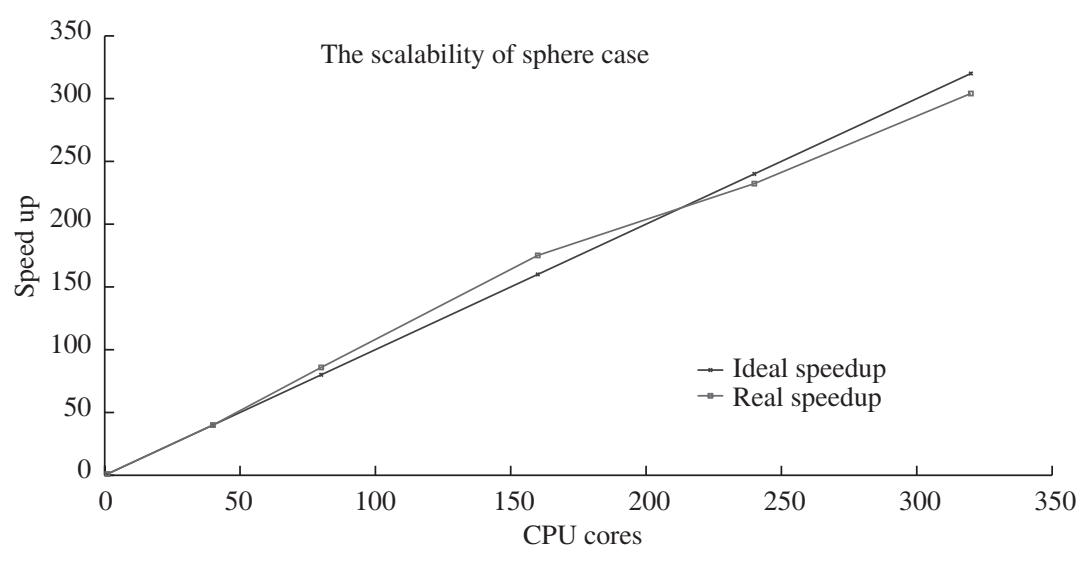

图 7 求解大球面面电荷密度算例的并行加速比

Figure 7 Speedup of solving the charge density on a big sphere

\section{4 基于有限元方法的光刻工艺仿真}

光刻工艺是集成电路制造工艺中的核心技术, 它通过光刻机系统, 实现了设计图形在半导体硅片 上的成像. 随着集成电路的制造工艺进入纳米时代, 芯片的特征尺寸减小到 $32 \mathrm{~nm}, 22 \mathrm{~nm}, 14 \mathrm{~nm}$ 以 至 $7 \mathrm{~nm}$, 但是高昂的研制费用使得光刻工艺依然采用 $193 \mathrm{~nm}$ 的大尺寸光波. 由于集成电路的器件和 互连线的特征尺寸比光源波长小, 光刻中由于大尺寸光波显著的干涉和衍射效应造成了实际在硅片上 成像的图形发生大尺寸图形扭曲变形, 小尺寸图形消失.

因此必须在集成电路设计制造过程中采用如光学邻近效应矫正 (OPC) 和移相掩膜 (PSM) 的分

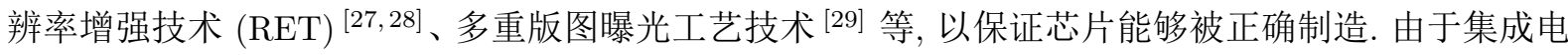
路光刻工艺极其昂贵和费时, 通过工艺试验的方法来获得实际光刻结果需要极其高昂的研发成本, 为 了保证芯片一次性流片成功, 必须对光刻工艺建模, 使得在芯片设计阶段就可以通过光刻模型来精确 地获得版图在硅片上成像的几何图形, 从而指导版图优化、掩膜矫正以及成品率分析.

图 8 是一个包含移相掩膜的光刻机系统示意图, 光从光源出发, 经过聚光器、移相掩膜版、光学 投影系统最终在硅片上成像. 值得注意的是, 当掩模版上的结构尺寸与光波波长相当或更小时, 光波 经过掩膜版将发生衍射、散射、极化等三维电磁现象. 传统的基于 Kirchhoff 几何光学近似的掩膜版模 型 ${ }^{[30]}$ 由于不能反映三维电磁现象, 不再适用, 因此必须采用严格的电磁场分析方法对移相掩膜版进 行精确建模.

针对集成电路光刻工艺仿真问题, 我们在并行自适应有限元平台 (PHG) 基础上发展了电磁散射 问题的有限元求解方法: 采用各向异性的单轴完美匹配层方法处理散射边界条件; 基于自适应有限 元计算框架, 设计自适应数值算法; 通过后验误差估计决定完全匹配层的厚度和其中的材料参数, 在 PML 层内解变化缓慢的区域自动产生较粗的网格, 使得总计算量对于层的厚度不敏感. 并行自适应算 法使得我们可以计算更加复杂和大规模的光刻仿真问题.

\section{1 光刻工艺仿真的散射模型}

光刻问题本质上可以看作三维电磁散射问题. 图 9 给出了一个简单的光刻仿真计算区域示例, 其 中 $\Omega_{2}$ 和 $\Omega_{3}$ 为光刻掩膜版区域, $\Omega_{2}$ 是透光的石英 (quartz) 材料, $\Omega_{3}$ 是不透光的铬 (chrome). 光波从 图 9(b) 上方入射, 穿过光刻掩膜版, 最终在硅片上的感光材料上成影. 我们关心光透过掩膜版后光场 


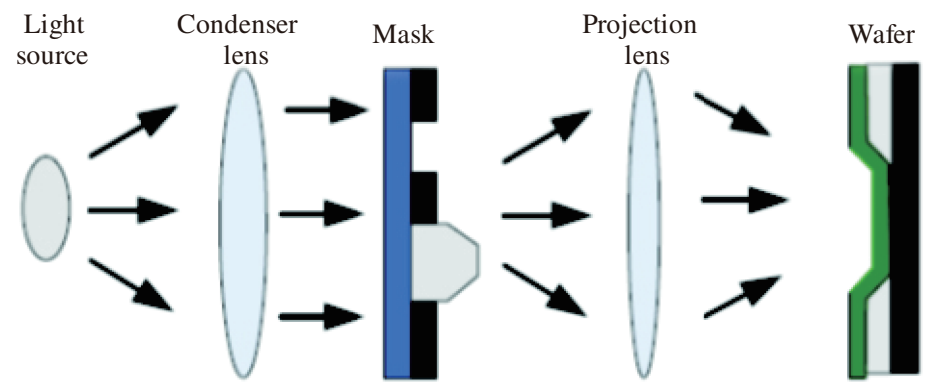

图 8 (网络版彩图) 光刻系统示意图

Figure 8 (Color online) Illustration of lithography system
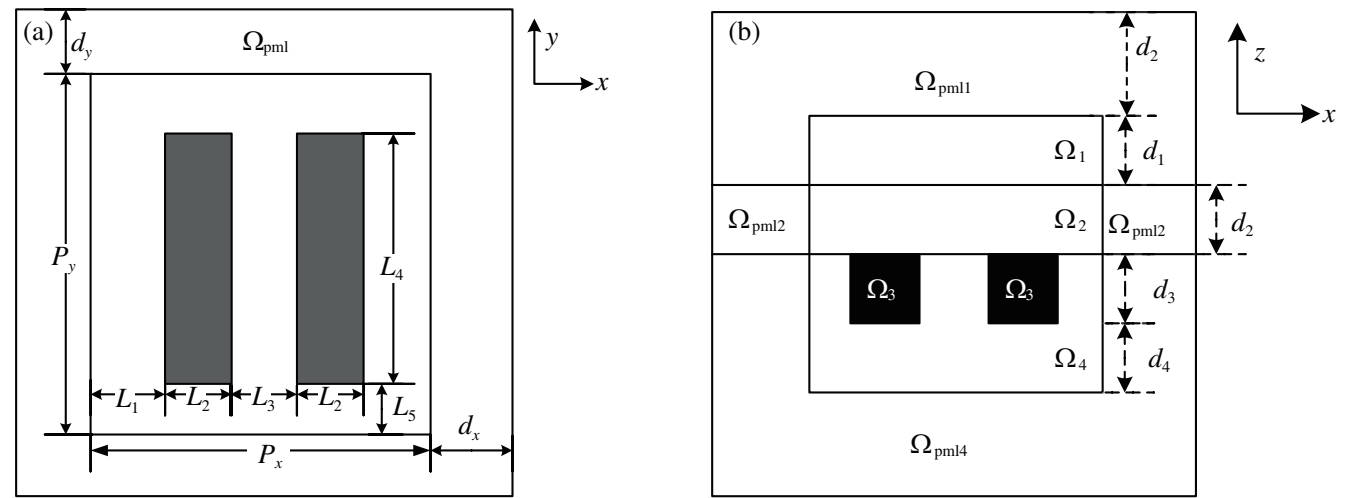

图 9 光刻仿真计算区域示例

Figure 9 Computation domain of lithography simulation, (a) $x-y$ plane; (b) $x$ - $z$ plane

强度的分布.

以图 9 为例, 光刻工艺仿真的散射问题可以定义为

$$
\left\{\begin{array}{l}
\nabla \times \nabla \times \boldsymbol{E}^{s}-k_{1}^{2} \boldsymbol{E}^{s}=0 \quad \text { in } \Omega_{1}, \\
\nabla \times \nabla \times \boldsymbol{E}^{s}-k_{2}^{2} \boldsymbol{E}^{s}=0 \quad \text { in } \Omega_{2}, \\
\nabla \times \nabla \times \boldsymbol{E}^{s}-k_{3}^{2} \boldsymbol{E}^{s}=\left(k_{3}^{2}-k_{4}^{2}\right) \boldsymbol{E}_{24}^{i} \quad \text { in } \Omega_{3}, \\
\nabla \times \nabla \times \boldsymbol{E}^{s}-k_{4}^{2} \boldsymbol{E}^{s}=0 \quad \text { in } \Omega_{4}, \\
r\left(\nabla \times \boldsymbol{E}^{s} \times \hat{\boldsymbol{x}}-\mathrm{i} k \boldsymbol{E}^{s}\right) \rightarrow 0 \quad \text { as } r=|x| \rightarrow \infty,
\end{array}\right.
$$

其中 $\boldsymbol{E}^{s}$ 为需求解的散射场, $D \subset R^{3}=\Omega_{1} \cup \Omega_{2} \cup \Omega_{3} \cup \Omega_{4}$ 是以 Lispschitz 多面体 $\Gamma_{D}$ 为边界的有界区 域. $\Omega_{1}$ 和 $\Omega_{4}$ 为空气, $\Omega_{2}$ 为石英材料, $\Omega_{3}$ 为铬材料.

假定计算区域中不包含铬材料, 即将 $\Omega_{3}$ 区域认为是空气, 得到图 10 所示计算区域. 若从 $\Omega_{1}$ 上 方入射的光波为 $\boldsymbol{E}_{1}^{i}=\boldsymbol{A}_{1} \mathrm{e}^{-\mathrm{i} k_{1} z}$, 则可以推导出从 $\Omega_{2}$ 入射 $\Omega_{4}$ 的光波 $\tilde{\boldsymbol{E}}_{24}^{i}$ :

$$
\tilde{\boldsymbol{E}}_{24}^{i}=\boldsymbol{A}_{4} \mathrm{e}^{-\mathrm{i} k_{4} z},
$$

其中

$$
\boldsymbol{A}_{4} \mathrm{e}^{\mathrm{i} k_{4}\left(d_{1}+d_{2}\right)}=T_{24} \boldsymbol{A}_{2} \mathrm{e}^{\mathrm{i} k_{2}\left(d_{1}+d_{2}\right)},
$$




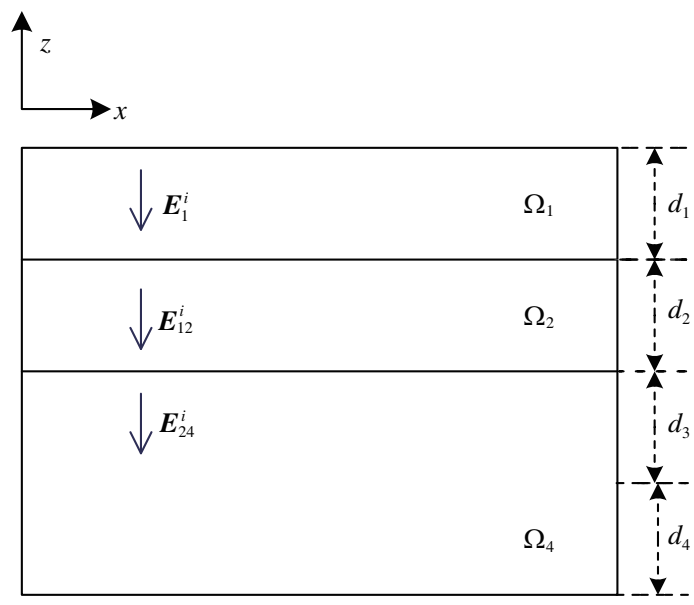

图 10 去除铬材料后的计算区域

Figure 10 The domain without chrome

$$
\begin{aligned}
& \boldsymbol{A}_{2} \mathrm{e}^{\mathrm{i} k_{2} d_{1}}=\boldsymbol{A}_{1} \mathrm{e}^{\mathrm{i} k_{1} d_{1}} \frac{T_{12}}{1-R_{21} R_{24} \mathrm{e}^{2 \mathrm{i} k_{2} d_{2}},}, \\
& R_{i j}=\frac{\mu_{j} k_{i}-\mu_{i} k_{j}}{\mu_{j} k_{i}+\mu_{i} k_{j}}, \quad(i, j=1,2,4), \\
& T_{i j}=\frac{2 \mu_{j} k_{i}}{\mu_{j} k_{i}+\mu_{i} k_{j}}, \quad(i, j=1,2,4) .
\end{aligned}
$$

假设式 (15) 中 $\boldsymbol{E}_{24}^{i}=\tilde{\boldsymbol{E}}_{24}^{i}$, 将其作为近似的光刻模拟散射模型.

\section{2 各向异性自适应完美匹配层方法}

针对电磁散射问题的有限元求解方法, 合理的边界条件设置对计算效率和精度有着决定性的影响. 我们采用各向异性的单轴完美匹配层方法处理散射边界条件, 如图 9, 记 $D$ 为立方体 $B=\{(x, y, z) \in$ $\left.R^{3}:|x|<\frac{P_{x}}{2}+d_{x},\left|x_{2}\right|<\frac{P_{y}}{2}+d_{y},\left|x_{3}\right|<\frac{P_{z}}{2}+d_{z}\right\}$ 的内部, $B=\Omega_{\mathrm{pml}} \cup D, \Omega_{\mathrm{pml}}=\Omega_{\mathrm{pml} 1} \cup \Omega_{\mathrm{pml} 2} \cup \Omega_{\mathrm{pml} 1}$, 得到对应于式 (15) 的完美匹配层方程:

$$
\left\{\begin{array}{l}
\nabla \times \boldsymbol{\Lambda} \nabla \times \boldsymbol{E}^{s}-\boldsymbol{\Lambda}^{-1} k_{1}^{2} \boldsymbol{E}^{s}=0 \quad \text { in } \Omega_{1} \cup \Omega_{\mathrm{pml1}}, \\
\nabla \times \boldsymbol{\Lambda} \nabla \times \boldsymbol{E}^{s}-\boldsymbol{\Lambda}^{-1} k_{2}^{2} \boldsymbol{E}^{s}=0 \quad \text { in } \Omega_{2} \cup \Omega_{\mathrm{pml} 2}, \\
\nabla \times \boldsymbol{\Lambda} \nabla \times \boldsymbol{E}^{s}-\boldsymbol{\Lambda}^{-1} k_{3}^{2} \boldsymbol{E}^{s}=\boldsymbol{\Lambda}^{-1}\left(k_{3}^{2}-k_{4}^{2}\right) \boldsymbol{E}_{24}^{i} \quad \text { in } \Omega_{3}, \\
\nabla \times \boldsymbol{\Lambda} \nabla \times \boldsymbol{E}^{s}-\boldsymbol{\Lambda}^{-1} k_{4}^{2} \boldsymbol{E}^{s}=0 \quad \text { in } \Omega_{4} \cup \Omega_{\mathrm{pml} 4}, \\
\boldsymbol{n} \times \boldsymbol{E}^{s}=0 \quad \text { on } \Gamma_{B},
\end{array}\right.
$$

其中

$$
\begin{gathered}
\boldsymbol{\Lambda}=\operatorname{diag}\left(\frac{\alpha_{1}}{\alpha_{2} \alpha_{3}}, \frac{\alpha_{2}}{\alpha_{3} \alpha_{1}}, \frac{\alpha_{3}}{\alpha_{1} \alpha_{2}}\right), \quad \alpha_{j}(t)=\eta_{j}+\mathrm{i} \sigma_{j}(t), \\
\sigma_{j}(t)=\tilde{\sigma}_{j}\left(\frac{|t|-\frac{L_{j}}{2}}{d_{j}}\right)^{2} \text { or } \sigma_{j}(t)=\tilde{\sigma}_{j}, \\
\eta_{j}= \begin{cases}1, & \left(\text { when } \sigma_{j} \leqslant 1\right), \\
1+\theta \sigma_{j}, & \left(\text { when } \sigma_{j}>1, \quad \theta \leqslant 1\right) .\end{cases}
\end{gathered}
$$


借鉴类似第 2 节中介绍的自适应有限元计算框架, 针对方程 (16), 设计自适应数值算法. 利用通过后 验误差估计决定完全匹配层的厚度和其中的材料参数, 在 PML 层内解变化缓慢的区域自动产生较粗 的网格, 使得总计算量对于层的厚度不敏感. 该自适应算法采用与第 2 节类似的网格局部加密策略. 针对方程 $(16)$ 的棱有限元数值解 $\boldsymbol{E}_{h}^{s}$, 提出任意的网格单元 $K$ 上的局部后验误差估计:

$$
\begin{aligned}
\eta_{K}^{2}= & h_{K}^{2}\left\|k^{2} \boldsymbol{\Lambda}^{-1} \boldsymbol{E}_{h}^{s}-\nabla \times\left(\boldsymbol{\Lambda} \nabla \times \boldsymbol{E}_{h}^{s}\right)\right\|_{L^{2}(K)}^{2} \\
& +h_{K}^{2}\left\|\nabla \cdot\left(k^{2} \boldsymbol{\Lambda}^{-1} \boldsymbol{E}_{h}^{s}\right)\right\|_{L^{2}(K)}^{2} \\
& +h_{K}\left\|\llbracket \boldsymbol{n} \times\left(\boldsymbol{\Lambda} \nabla \times \boldsymbol{E}_{h}^{s}\right) \rrbracket\right\|_{L^{2}(\partial K)}^{2}+h_{K}\left\|\llbracket k^{2} \boldsymbol{\Lambda}^{-1} \boldsymbol{E}_{h}^{s} \cdot \boldsymbol{n} \rrbracket\right\|_{L^{2}(\partial K)}^{2},
\end{aligned}
$$

其中 $h_{K}$ 为 $K$ 的直径, 『·为单元面上的跳量, $\boldsymbol{n}$ 为单元面上的外法向.

\section{3 数值结果}

本文通过图 9 算例验证自适应完美匹配层方法的有效性和正确性, 其中图 9 两根连线版图对应 的铬材料尺寸是 $100 \mathrm{~nm} \times 1000 \mathrm{~nm} \times 50 \mathrm{~nm}$, 间距 $200 \mathrm{~nm}$, 因此, $L_{1}=100 \mathrm{~nm}, L_{2}=100 \mathrm{~nm}, L_{3}=$ $200 \mathrm{~nm}, L_{4}=1000 \mathrm{~nm}, d_{1}=d_{4}=100 \mathrm{~nm}, d_{2}=d_{3}=50 \mathrm{~nm}, P_{x}=600 \mathrm{~nm}, P_{y}=1200 \mathrm{~nm}, P_{z}=300 \mathrm{~nm}$. 入射光波波长设为 $193 \mathrm{~nm}$, 其他相关物理参数:

$$
\begin{gathered}
k_{i}^{2}=s^{2} \omega^{2} \epsilon_{i} \mu_{i}, \quad(i=1,2,3,4) ; \\
\mu_{i}=\mu_{0}=4 \pi \times 10^{-7} \mathrm{Hm}^{-1}, \quad(i=1,2,3,4) ; \\
\epsilon_{0}=8.854 \times 10^{-12} \mathrm{Fm}^{-1}, \\
\epsilon_{1}=\epsilon_{4}=\epsilon_{0}, \epsilon_{2}=2.25 \epsilon_{0}, \epsilon_{3}=(2.25+10 \mathrm{i}) \epsilon_{0} .
\end{gathered}
$$

计算程序基于并行自适应有限元平台 (PHG) 实现, 离散问题方程组求解采用 MPI 和 OpenMP 混合并行的稀疏直接法求解器 MUMPS ${ }^{[31]}$. 计算平台为中国科学院科学与工程计算国家重点实验室 的 LSSC-III 机群. 直接法求解器对内存资源要求很高, 我们采用 MPI 和 OpenMP 混合并行的方式, 在每个计算结点 (8 个 CPU 核) 启动一个 MPI 进程, 每个 MPI 进程管理 8 个 OpenMP 线程.

图 11 给出了误差收玫曲线, 即 $\log N-\log \mathcal{E}$ 曲线, 其中 $\mathcal{E}$ 是后验误差估计值, $N$ 是自由度. 图 11 验证了自适应有限元中网格自适应加密的最优收玫特性, 即 $\mathcal{E} \approx C N^{-1 / 3}$.

本文针对图 12 的存储器 (RAM) 互连电路版图算例进行强可扩展性测试. 该版图算例包含 10 个导体的版图图形, 通过采用 $\mathrm{OPC}$ 技术对版图图形进行校正以增加光刻的分辨率, 计算区域大小为 $5160 \mathrm{~nm} \times 3000 \mathrm{~nm} \times 800 \mathrm{~nm}$, PML 层厚度选为 $200 \mathrm{~nm}$. 计算实验的未知量个数为 11367392 , 分别采 用 32 节点 (256 CPU 核), 64 节点 (512 CPU 核) 和 128 节点 (1024 CPU 核) 计算, 该算例由于内存用 量大, 至少需要 32 节点才能计算, 因此我们使用 32 节点 $256 \mathrm{CPU}$ 核的计算时间作为并行效率的测试 基准. 如表 4 所示, 在使用 512 核和 1024 核时, 组装线性系统的效率分别为 $94.7 \%$ 和 $98.3 \%$, 接近线 性的扩展性, 而求解线性系统即离散方程组的效率略差, 分别为 $67.5 \%$ 和 $49.2 \%$, 主要受稀疏直接法的 扩展性的制约.

图 13 给出了计算得到的光强分布, 图 13(a) 为 $z=-10 \mathrm{~nm}$ 处光强分布, 图 13(b) 为 $z=-50 \mathrm{~nm}$ 处光强分布, 通过图 13 可以看到光场通过掩膜版后产生了干涉现象. 因此, 基于并行自适应有限元的 光刻仿真方法可以用于光学临近校正 $(\mathrm{OPC})$ 、移相掩模 $(\mathrm{PSM})$ 、多重曝光等工艺的版图分析和缺陷 分析. 


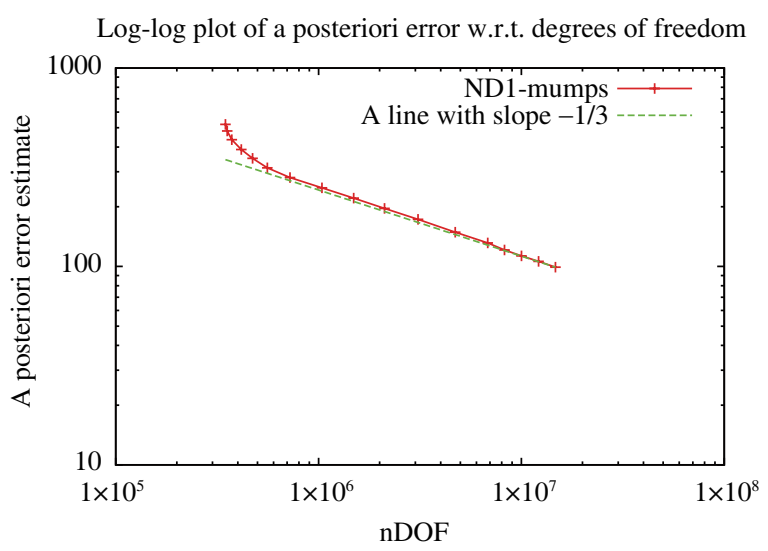

图 11 (网络版彩图) 自适应网格加密的最优收敛特性

Figure 11 (Color online) The quasi-optimality of the adaptive mesh refinements

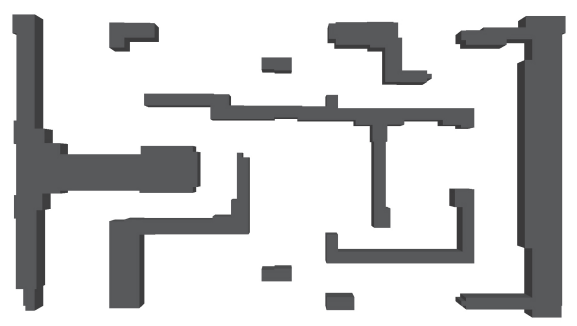

图 12 OPC 校正的存储器 (RAM) 互连电路版图算例

Figure 12 RAM interconnect mask example with OPC

表 4 存储器 (RAM) 互连电路版图的并行效率测试

Table 4 Parallel efficiency of RAM interconnect mask example

\begin{tabular}{|c|c|c|c|c|c|c|c|}
\hline \multirow{2}{*}{$\begin{array}{l}\text { \#MPI } \\
\text { threads }\end{array}$} & \multirow{2}{*}{$\begin{array}{c}\# \mathrm{CPU} \\
\text { cores }\end{array}$} & \multicolumn{2}{|c|}{ Assembling } & \multicolumn{2}{|c|}{ Solving } & \multirow{2}{*}{$\begin{array}{c}\text { Overall } \\
\text { efficiency (\%) }\end{array}$} & \multirow{2}{*}{$\begin{array}{c}\text { Memory cost } \\
\text { per node (GB) }\end{array}$} \\
\hline & & Time (s) & Efficiency (\%) & Time (s) & Efficiency (\%) & & \\
\hline 32 & $32 \times 8=256$ & 3.58 & - & 5356 & - & - & 20.8 \\
\hline 64 & $64 \times 8=512$ & 1.89 & 94.7 & 3966 & 67.5 & 67.5 & 12.7 \\
\hline 128 & $128 \times 8=1024$ & 0.91 & 98.3 & 2722 & 49.2 & 49.2 & 7.2 \\
\hline
\end{tabular}

\section{MPI 和 OpenMP 混合并行及并行性讨论}

本文介绍了纳米集成电路互连线建模和光刻仿真中大规模复杂介质环境 Maxwell 方程数值求解 的 3 个并行计算应用实例, 通过采用 MPI 和 OpenMP 混合并行的编程模式, 可以在大规模高性能计 算机集群系统上实现互连线建模和光刻仿真的高效求解.

MPI 和 OpenMP 是实现并行计算的两种主流方式: MPI 通过进程间消息传递的机制实现进程级 的并行计算, 可以实现跨计算节点的大规模并行; OpenMP 采用共享内存的机制实现线程级的并行计 算, 主要实现计算节点内部并行. MPI 并行效率受限于集群系统的通讯网络性能、算法的通讯复杂度 等. 对于具有较高通讯复杂度的算法, 当 MPI 进程数过大时, 往往导致 MPI 并行效率的急剧下降, 在 表 2 中可以发现随着进程数增多, 并行效率下降的情况. OpenMP 并行效率受限于访存性能、算法复 

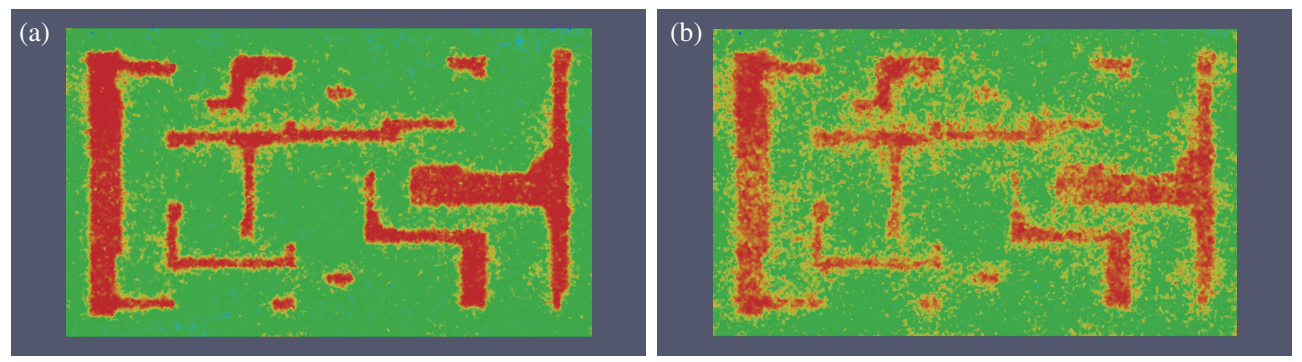

图 13 (网络版彩图) 存储器 (RAM) 互连电路版图的光强分布结果

Figure 13 (Color online) The magnitude of the electric field in RAM interconnect mask example. (a) $z=-10 \mathrm{~nm}$ plane, (b) $z=-50 \mathrm{~nm}$ plane

表 5 OpenMP 并行效率测试 [9]

Table 5 Parallel Scalability of OpenMP [9]

\begin{tabular}{cccc}
\hline \#CPU cores & Computation time $(\mathrm{s})$ & Speedup & Parallel efficiency $(\%)$ \\
\hline 1 & 2275.20 & $1 \mathrm{X}$ & 100 \\
2 & 1500.61 & $1.52 \mathrm{X}$ & 75.81 \\
3 & 1210.15 & $1.88 \mathrm{X}$ & 62.67 \\
4 & 1025.79 & $2.22 \mathrm{X}$ & 55.45 \\
6 & 1005.96 & $2.26 \mathrm{X}$ & 37.70 \\
8 & 1007.79 & $2.26 \mathrm{X}$ & 28.22 \\
\hline
\end{tabular}

杂度等, 其 OpenMP 并行粒度相对小于 MPI 并行粒度. 现有的高性能计算机, 每个计算节点有 2 到 4 个处理器, 每个处理器最高有 24 个 CPU 核, 多层的内存结构使得不同的 CPU 核访问相同内存具有很 大的差异. 对于具有较高访存复杂度的算法, 当 OpenMP 线程数太大时, 导致 OpenMP 并行效率急剧 下降. 以寄生电容提取问题为例, 表 5 给出了单个计算节点 OpenMP 并行效率的测试结果, OpenMP 的并行效率随着 CPU 核数增加而递减. 在该测试算例中, 当 CPU 核数为 4 时, OpenMP 具有 $55 \%$ 的 并行效率, 当 $\mathrm{CPU}$ 数为 6 时, OpenMP 的并行加速比达到饱和.

一般来说，对于具有较高复杂度的算法，在使用数十上百处理器核时 MPI 并行的效率高于 OpenMP 效率, 可以采用纯 MPI 并行. 但是在超大规模高性能计算机集群中, 存在成千上万个计 算节点, 数十上百万个 CPU 核, 对于大规模高性能计算机集群的并行仿真计算, 需要采用 MPI 和 OpenMP 的混合并行模式. 当继续增加进程数带来的 MPI 并行加速比低于通过增加线程数带来的 OpenMP 加速比时, 可以采用 MPI 和 OpenMP 的混合并行模式, 通过 OpenMP 辅助 MPI 使得保证 一定并行效率下可以使用更多的 CPU 核. 例如在电容参数提取问题中, 当单纯使用 MPI 并行的并行 效率低于 $60 \%$ 时, 可以采用 MPI 和 OpenMP 混合模式, 每个 MPI 进程管理 4 6 个 OpenMP 线程仍 可进一步增加 CPU 核数获得较好的并行效率. 但是, 若使用超过 6 个 OpenMP 线程, 多余的线程也 不会带来额外的加速.

大规模集群并行计算的并行效率与具体的问题及相应的求解算法密切相关. 在 Maxwell 方程求解 的各种常用方法中, 与边界元方法相比, 有限元方法采用局部基对方程进行求解, 通过采用适当的分布 式网格管理可以保证较低的进程间通信, 因此, 在大规模并行计算中, 有限元方法往往比边界元方法 具有更好的并行效率. 在本文综述的 3 个问题及算法中, 随机行走方法适用于求解静电场问题, 由于 每次随机行走之间相对独立, 对 CPU 核间的通信要求极低, 十分适用于超大规模计算集群并行计算. 
相对于互连线寄生参数提取和光刻仿真采用有限元方法而言, 基于随机行走方法的 BIE-WOS 算法在 表面电荷计算问题的应用中取得了较高的并行效率.

\section{6 总结}

集成电路互连线建模和光刻工艺仿真涉及大规模复杂介质环境 Maxwell 方程的数值求解, 计算复 杂度高、规模庞大, 发展大规模并行计算方法是解决计算瓶颈问题的有效途径. 本文主要综述纳米集 成电路互连线建模和光刻仿真中的大规模并行计算方法. 首先, 综述了寄生电容提取的并行自适应有 限元方法 ParAFEMCap, 在国家超算天津中心的天河 1A 并行计算机 1536 CPU 核下完成了测试, 具 有 $75.7 \%$ 的并行效率; 其次, 综述了结合边界元法与随机法的互连线粗粘表面电荷分布计算方法, 本 文进一步在 5120 核上验证了算法近似线性的并行加速比. 在光刻仿真方面, 基于自适应有限元计算框 架 (PHG) 提出了集成电路光刻的并行自适应仿真方法, 采用各项异性的单轴完美匹配层方法处理散 射边界条件, 实验结果表明自适应网格加密具有最优收玫特性, 可以精确模拟光刻中产生的图形畸变 现象. 在未来, 将继续基于陈志明教授等提出的波源转移区域分解算法 ${ }^{[32]}$ 发展可扩展高效的求解光 刻离散问题的迭代算法, 提高光刻模拟的并行效率.

\section{参考文献}

1 Sabelka R, Harlander C, Selberherr S. The state of the art in interconnect simulation. In: Proceedings of International Conference on Simulation of Semiconductor Processes and Devices, Seattle, 2000. 6-11

2 Nagaraj N S, Bonifield T, Singh A, et al. Benchmarks for interconnect parasitic resistance and capacitance. In: Proceedings of the 4th International Symposium on Quality Electronic Design, San Jose, 2003. 163-168

3 Kao W H, Lo C, Basel M, et al. Parasitic extraction: current state of the art and future trends. Proc IEEE, 2001, 89: 729-739

4 Delorme N, Belleville M, Chilo J. Inductance and capacitance analytic formulas for vlsi interconnects. Electron Lett, 1996, 32: 996-997

5 Nabors K, White J. FastCap: a multipole accelerated 3-D capacitance extraction program. IEEE Trans Comput-Aided Design Integr Circ Syst, 1991, 10: 1447-1459

6 Yan S, Sarin V, Shi W. Sparse transformations and preconditioners for 3-D capacitance extraction. IEEE Trans Comput-Aided Design Integr Circ Syst, 2005, 24: 1420-1426

7 Shi W, Liu J, Kakani N, et al. A fast hierarchical algorithm for three-dimensional capacitance extraction. IEEE Trans Comput-Aided Design Integr Circ Syst, 2002, 21: 330-336

8 Ozgun O, Mittra R, Kuzuoglu M. CBFEM-MPI: a parallelized version of characteristic basis finite element method for extraction of 3-D interconnect capacitances. IEEE Trans Adv Packag, 2009, 32: 164-174

9 Chen G L, Zhu H L, Cui T, et al. Parafemcap: a parallel adaptive finite-element method for 3-D VLSI interconnect capacitance extraction. IEEE Trans Microw Theory Tech, 2012, 60: 218-231

10 Veremey V, Mittra R. A technique for fast calculation of capacitance matrices of interconnect structures. IEEE Trans Compon Packag Manuf Tech Part B: Adv Packag, 1998, 21: 241-249

11 Coz Y L L, Iverson R B. A stochastic algorithm for high speed capacitance extraction in integrated circuits. Solid-State Electron, 1992, 35: 1005-1012

12 Yuan Y, Banerjee P. A parallel implementation of a fast multipole-based 3-D capacitance extraction program on distributed memory multicomputers. J Parallel Distrib Comput, 2001, 61: 1751-1774

13 Zhao X Q, Feng Z. Fast multipole method on gpu tackling 3-D capacitance extraction on massively parallel simd platforms. In: Proceedings of IEEE/ACM Design Automation Conference, New York, 2011. 558-563

14 Hsiao Y C, Daniel L. Caplet: a highly parallelized field solver for capacitance extraction using instantiable basis functions. IEEE Trans Comput-Aided Design Integr Circ Syst, 2016, 35: 458-470 
15 Yan C H, Cai W, Zeng X. A parallel method for solving laplace equations with dirichlet data using local boundary integral equations and random walks. SIAM J Sci Comput, 2013, 35: B868-B889

16 Wong A K, Neureuther A R. Rigorous three-dimensional time-domain finite-difference electromagnetic simulation for photolithographic applications. IEEE Trans Semicond Manuf, 1995, 8: 419-431

17 Lucas K D, Tanabe H, Strojwas A J. Effcient and rigorous three-dimensional model for optical lithography simulation. J Opt Soc America, 1996, 13: 2187-2199

18 Cai W, Ji X, Sun J, et al. A schwarz generalized eigen-oscillation spectral element method (GeSEM) for 2-D high frequency electromagnetic scattering in dispersive inhomogeneous media. J Comput Phys, 2008, 227: 9933-9954

19 Zong K, Zeng X, Ji X, et al. Highly parallel rigorous simulation of phase shift masks with a generalized eigen-oscillation spectral element method (GeSEM). J Micro/Nanolith MEMS MOEMS (JM3), 2009, 8: 031403

20 Burger S, Kohle R, Zschiedrich L, et al. Benchmark of FEM, waveguide and FDTD algorithms for rigorous mask simulation. Proc SPIE, 2005, 5992: 368-379

21 Chen Z, Dai S. On the efficiency of adaptive finite element methods for elliptic problems with discontinuous coefficients. SIAM J Sci Comput, 2002, 24: 443-462

22 Zhang L B, Zheng W Y, Lu B Z, et al. The toolbox PHG and its applications. Sci Sin Inform, 2016, 10: 1442-1464 [张 林波, 郑伟英, 卢本卓, 等. 并行自适应有限元软件平台 PHG 及其应用. 中国科学: 信息科学, 2016, 10: 1442-1464]

23 Cui T, Chen J Q, Zhu H L, et al. Algorithms in ParAFEMImp: a parallel and wideband impedance extraction program for complicated 3-D geometries. In: Proceedings of IEEE International Conference on High Performance and Smart Computing (HPSC), New York, 2016. 304-309

24 Zhang L. A parallel algorithm for adaptive local refinement of tetrahedral meshes using bisection. Numer Math Theory Meth Appl, 2009, 2: 65-89

25 Friedman A. Stochastic Differential Equations and Applications. New York: Dover Publications, 2006

26 Hwang C O, Given J A. Last-passage monte carlo algorithm for mutual capacitance. Phys Rev E, 2006, 74: 027701

27 Wong A S, Newmark D M, Rolfson J B, et al. Investigating phase-shifting mask layout issues using a CAD toolkit. In: Proceedings of International Electron Devices Meeting, Washington, 1991. 705-708

28 Yu P, Pan D Z. TIP-OPC: a new topological invariant paradigm for pixel based optical proximity correction. In: Proceedings of IEEE/ACM International Conference on Computer-Aided Design, San Jose, 2007. 847-853

29 Pan D Z, Liebmann L, Yu B, et al. Pushing multiple patterning in sub-10nm: are we ready? In: Proceedings of the 52nd IEEE/ACM Design Automation Conference (DAC), San Francisco, 2015. 1-6

30 Ponomarev G A, Yakubov V P. Kirchhoff's approximation method of geometrical optics in the problem of scattering of waves at an irregular surface. Radiophys Quant Electron, 1976, 19: 849-852

31 Amestoy P R, Duff I S, L'Excellent J Y, et al. A fully asynchronous multifrontal solver using distributed dynamic scheduling. Siam J Matrix Anal Appl, 2001, 23: 15-41

32 Chen Z M, Xiang X S. A source transfer domain decomposition method for helmholtz equations in unbounded domain. SIAM J Numer Anal, 2013, 51: 2331-2356

\title{
Massively parallel computing in nano-VLSI interconnect modeling and lithography simulation
}

\author{
Hengliang ZHU ${ }^{1}$, Xuan ZENG ${ }^{1 *}$, Tao CUI $^{2}$, Changhao YAN ${ }^{1} \&$ Linbo ZHANG ${ }^{2}$ \\ 1 State Key Laboratory of ASIC and System, Fudan University, Shanghai 201203, China; \\ 2 State Key Laboratory of Scientic and Engineering Computing, Academy of Mathematics and Systems Science, \\ Chinese Academy of Sciences, Beijing 100190, China \\ *E-mail: xzeng@fudan.edu.cn
}

\begin{abstract}
VLSI is large in scale, and complex in structure. In today's nano-VLSI, serious process variations induced by the complex nanometer integrated circuit process technology may result in severe degradation of the
\end{abstract}


integrated circuit performance. These factors present ever increasing challenges for present day nano-scale VLSI design. Interconnect modeling and lithography simulations rely on numerical approaches for solving large-scale Maxwell's equations, of which the computational cost is extremely high. In this paper, several massively parallel computing approaches for interconnect modeling and lithography simulation are surveyed, based on adaptive finite element theory and a parallel hierarchical grid (PHG) platform. Regarding interconnect modeling, we first review the parallel adaptive finite-element method ParAFEMCap for parasitic capacitance extraction, which achieves a parallel efficiency of $75.7 \%$ on $1536 \mathrm{CPU}$ cores. In addition, we review a hybridization of the boundary integral equation method and the random walk on spheres method (BIE-WOS) for surface charge density computations for conductors or dielectric mediums. The proposed method proves to be superior to existing methods for massively parallel computing. On a supercomputer with $5120 \mathrm{CPU}$ cores, BIE-WOS can achieve almost a linear parallel efficiency. Regarding lithography simulation, we propose a parallel adaptive finite-element framework method by adopting the PHG (parallel hierarchical grid) platform and a perfectly matched anisotropy uniaxial layer to handle scattering boundary conditions.

Keywords VLSI, modeling, parasitic extraction, lithography, finite element method, random walk

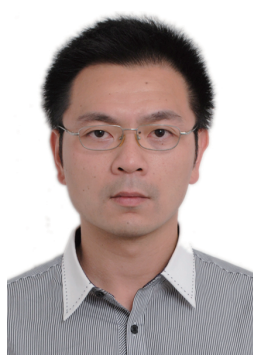

Hengliang ZHU was born in 1982. He received the B.E. degree in electronic engineering from the University of Science and Technology of China in 2004 and the Ph.D. degree in microelectronics from Fudan University in 2009. He joined the State Key Laboratory of Application Specific Integrated Circuits (ASIC) and Systems, Microelectronics Department at Fudan University as an assistant professor in 2009. His research interests include circuit analysis, interconnect parameter extraction, and model order reduction.

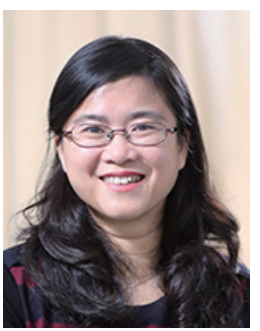

Xuan ZENG was born in 1969. She received B.S. and Ph.D. degrees in electrical engineering from Fudan University, Shanghai, China, in 1991 and 1997, respectively. She is currently a full professor with the Department of Microelectronics, Fudan University. She was a visiting professor with the Department of Electrical Engineering, Texas A\&M University, USA, and the Department of Microelectronics, Technische Universiteit Delft, The Netherlands, in 2002 and 2003, respectively. Her current research interests include design for manufacturability, high-speed interconnect analysis and optimization, and circuit simulation.

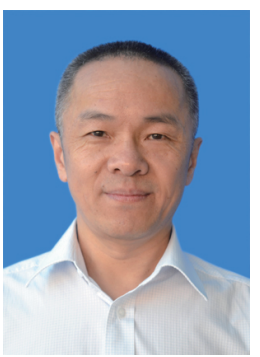

Linbo ZHANG was born in 1962. He received his $\mathrm{Ph} . \mathrm{D}$. degree in Mathematics from Université de Paris-sud, France, in 1987. Currently, he is a professor of the Academy of Mathematics and Systems Science of the Chinese Academy of Sciences, and the directory of State Key Laboratory of Scientific and Engineering Computing. His research interests include numerical algorithms and high performance computing. 